\title{
Three\#dimensional Magnetohydrodynamic Simulations of Spherical Accretion
}

\section{Citation}

Igumenshchev, Igor V., and Ramesh Narayan. 2002. "Three\#dimensional Magnetohydrodynamic Simulations of Spherical Accretion." The Astrophysical Journal 566 (1): 137-47. https:// doi.org/10.1086/338077.

\section{Permanent link}

http://nrs.harvard.edu/urn-3:HUL.InstRepos:41384947

\section{Terms of Use}

This article was downloaded from Harvard University's DASH repository, and is made available under the terms and conditions applicable to Other Posted Material, as set forth at http:// nrs.harvard.edu/urn-3:HUL.InstRepos:dash.current.terms-of-use\#LAA

\section{Share Your Story}

The Harvard community has made this article openly available.

Please share how this access benefits you. Submit a story. 
The Astrophysical Journal, 566:137-147, 2002 February 10

(C) 2002. The American Astronomical Society. All rights reserved. Printed in U.S.A.

\title{
THREE-DIMENSIONAL MAGNETOHYDRODYNAMIC SIMULATIONS OF SPHERICAL ACCRETION
}

\author{
IGOR V. IGUMENSHCHEV ${ }^{1,2}$ AND RAMESH NARAYAN \\ Harvard-Smithsonian Center for Astrophysics, 60 Garden Street, Cambridge, MA 02138 \\ Received 2001 May 19; accepted 2001 October 15
}

\begin{abstract}
We present three-dimensional numerical magnetohydrodynamic simulations of radiatively inefficient spherical accretion onto a black hole. The simulations are initialized with a Bondi flow and with a weak, dynamically unimportant, large-scale magnetic field. As the gas flows in, the magnetic field is amplified. When the magnetic pressure approaches equipartition with the gas pressure, the field begins to reconnect and the gas is heated up. The heated gas is buoyant and moves outward, causing line stretching of the frozen-in magnetic field. This leads to further reconnection and more heating and buoyancy-induced motions, so that the flow makes a transition to a state of self-sustained convection. The radial structure of the flow changes dramatically from its initial Bondi profile, and the mass accretion rate onto the black hole decreases significantly. Motivated by the numerical results, we develop a simplified analytical model of a radiatively inefficient spherical flow in which convective transport of energy to large radii plays an important role. In this "convection-dominated Bondi flow," the accretion velocity is highly subsonic, and the density varies with radius as $\rho \propto R^{-1 / 2}$ rather than the standard Bondi scaling $\rho \propto R^{-3 / 2}$. We estimate that the mass accretion rate onto the black hole correspondingly scales as $\dot{M} \sim$ $\left(R_{\text {in }} / R_{a}\right) \dot{M}_{\text {Bondi }}$, where $R_{\text {in }}$ is a small multiple of the Schwarzschild radius of the black hole and $R_{a}$ is the "accretion radius" at which the ambient gas in the surrounding medium is gravitationally captured by the black hole. Since the factor $R_{\text {in }} / R_{a}$ is typically very small, $\dot{M}$ is significantly less than the Bondi accretion rate. Convection-dominated Bondi flows may be relevant for understanding many astrophysical phenomena, e.g., post-supernova fallback and radiatively inefficient accretion onto supermassive black holes, stellar-mass black holes, and neutron stars.

Subject headings: accretion, accretion disks - convection — galaxies: nuclei - MHD supernovae: general - turbulence
\end{abstract}

\section{INTRODUCTION}

The classic problem of adiabatic spherical accretion onto a compact gravitating mass has been studied by many authors. The hydrodynamic version of this problem was solved by Bondi (1952), who showed that inside a certain capture radius, the radial velocity of the accreting gas varies as $v_{R} \propto R^{-1 / 2}$, where $R$ is the radius. Correspondingly, the density varies as $\rho \propto R^{-3 / 2}$.

If the gas in a Bondi flow has a frozen-in magnetic field, the field lines are stretched in the radial direction and compressed in the transverse direction, so that the radial component of the field is amplified according as $B_{R} \propto R^{-2}$. The magnetic energy density then varies as $\epsilon_{m}=B^{2} / 8 \pi \propto R^{-4}$. However, the gravitational energy density of the gas varies only as $\epsilon_{\text {grav }}=\rho G M / R \propto R^{-5 / 2}$. Thus, for a sufficiently small radius, one formally has $\epsilon_{m} \gg \epsilon_{\text {grav }}$, which is physically inconsistent since the magnetic energy ultimately is derived from the gravitational binding energy of the accreting gas.

Shvartsman (1971) proposed that the conversion of gravitational energy into magnetic energy is accompanied by turbulence, which tangles the magnetic field lines. He suggested that, as the magnetic and gravitational energies approach equipartition, field reconnection ensures that $\epsilon_{m}$ does not exceed $\epsilon_{\text {grav }}$. Reconnection will be accompanied by dissipation (Bisnovatyi-Kogan \& Ruzmaikin 1974; Mészáros 1975), which will heat the gas. As a result, the thermal energy of the gas is also likely to come into rough

\footnotetext{
${ }^{1}$ Laboratory for Laser Energetics, University of Rochester, 250 East River Road, Rochester, NY 14623-1299.

${ }^{2}$ Institute of Astronomy, 48 Pyatnitskaya Ulitsa, 109017 Moscow,
}

equipartition with the other energies. These modifications have been recognized for many years and have been incorporated into models of spherical accretion flows.

To our knowledge, all published studies of the magnetized spherical accretion problem have assumed that the magnetic field causes no serious dynamical effects on the flow. In particular, all authors assume that the velocity and density scale exactly as in the Bondi solution, namely, $v_{R} \propto$ $R^{-1 / 2}, \rho \propto R^{-3 / 2}$. (The following is an incomplete list of relevant papers on the subject: Zeldovich \& Novikov 1971; Shapiro 1973a, 1973b; Ipser \& Price 1977, 1982, 1983; Maraschi et al. 1979; Maraschi, Roasio, \& Treves 1982; Scharlemann 1981, 1983; Treves, Maraschi, \& Abramowicz 1988; Turolla \& Nobili 1989; Nobili, Turolla, \& Zampieri 1991; Mason \& Turolla 1992; Mason 1992; Melia 1992; Kowalenko \& Melia 1999; Coker \& Melia 2000. Many other papers discuss spherical accretion without explicitly considering magnetic fields and heating, e.g., Ostriker et al. 1976; Park \& Ostriker 1989; Houck \& Chevalier 1991; Zampieri et al. 1998.)

In this paper we show that the magnetic field can play an important, perhaps even dominant, role in the dynamics of spherical accretion flows. The influence is both direct, through the action of the electromotive force, and indirect, through the entropy generated in the process of field reconnection. The latter leads to convection, which drastically changes the flow structure relative to the Bondi solution. In particular, the density profile becomes much less centrally peaked than the Bondi $R^{-3 / 2}$ profile, and the mass accretion rate is reduced significantly below the Bondi rate. Interestingly, the flow resembles the recently discovered hydrodynamic convection-dominated accretion flow solution (CDAF; Narayan, Igumenshchev, \& Abramowicz 
2000; Quataert \& Gruzinov 2000; Igumenshchev \& Abramowicz 1999, 2000, 2001; Stone, Pringle, \& Begelman 1999; Igumenshchev, Abramowicz, \& Narayan 2000; Ball, Narayan, \& Quataert 2001).

The paper is organized as follows: In $\S 2$ we describe our numerical method and the details of our initial and boundary conditions. In $\S 3$ we present results of numerical simulations, and in $\S 4$ we describe an approximate self-similar solution that includes the effects of plasma heating and convection. In $\S 5$ we discuss some implications of the results, and in $\S 6$ we conclude with a summary.

\section{SIMULATION TECHNIQUE}

\subsection{Magnetohydrodynamic Equations}

We solve the equations of resistive MHD in the one-fluid approximation,

$$
\begin{gathered}
\frac{d \rho}{d t}+\rho \nabla \cdot v=0, \\
\rho \frac{d v}{d t}=-\nabla(P+Q)-\rho \nabla \Phi+\frac{1}{4 \pi}(\nabla \times B) \times B, \\
\rho \frac{d \epsilon}{d t}=-(P+Q) \nabla \cdot v+\frac{1}{4 \pi} \eta J^{2}, \\
\frac{\partial \boldsymbol{B}}{\partial t}=\nabla \times(v \times B-\eta \boldsymbol{J}),
\end{gathered}
$$

where $\rho$ is the density, $v$ is the velocity, $P$ is the pressure, $\Phi$ is the gravitational potential, $B$ is the magnetic induction, $\epsilon$ is the specific internal energy, $\boldsymbol{J}=\boldsymbol{\nabla} \times \boldsymbol{B}$ is the current density, and $\eta$ is the resistivity. The terms involving $Q$ in equations (2) and (3) correspond to an artificial viscosity that is introduced to correctly treat shocks. We adopt the ideal gas equation of state,

$$
P=(\gamma-1) \rho \epsilon,
$$

with an adiabatic index $\gamma=5 / 3$. We assume that there is no radiative cooling.

We take the compact mass at the center to be a black hole of mass $M$, and we use a pseudo-Newtonian gravitational potential (Paczyński \& Wiita 1980) to mimic the effects of general relativity,

$$
\Phi=-\frac{G M}{R-R_{g}}, \quad R_{g}=\frac{2 G M}{c^{2}},
$$

where $R_{g}$ is the gravitational radius.

\subsection{Numerical Method}

We numerically solve equations (1)-(4) by using an extension of the original PPM algorithm developed by Colella \& Woodward (1984) for hydrodynamics. We use the Lagrangian version of the PPM algorithm with operator splitting. The Riemann solver takes into account the nonlinear interaction of the fast MHD waves when calculating time updates of the density, velocity and internal energy in equations (1)-(3). The components of the Lorentz force in the equation of motion (2) are calculated using a solution of the Lagrangian characteristic equations for Alfvén waves, as in the method of characteristics (MOC) of Stone \& Norman
(1992). We use dimension splitting when solving the equations in three dimensions.

We replace the induction equation (4) by an equivalent equation for the vector potential $\boldsymbol{A}$,

$$
\frac{\partial \boldsymbol{A}}{\partial t}=\boldsymbol{v} \times \boldsymbol{B}-\eta \boldsymbol{J}
$$

where $\boldsymbol{B}=\boldsymbol{\nabla} \times \boldsymbol{A}$. This approach guarantees that the constraint $\boldsymbol{\nabla} \cdot \boldsymbol{B}=0$ is satisfied to within grid approximation errors at each time $t$. The first term on the right-hand side of equation (7) is calculated using a modified version of the Eulerian MOC algorithm (Stone \& Norman 1992). (The modification is due to our use of a different representation of the location of the Alfvén characteristics domain for calculating the averaged values of the components of $v$ and $\boldsymbol{B}$.)

The code operates on a three-dimensional Cartesian grid. In order to adequately resolve the large dynamic range of spatial scales spanned by astrophysical accretion flows, we employ a nested grid (see Fig. 1) similar to that employed by Igumenshchev et al. (2000) for simulating hydrodynamic CDAFs in three dimensions. In the present calculations, we have used five subgrids with $64 \times 64 \times 64$ cells in each subgrid. The cell size in the innermost subgrid is $\Delta_{1}=$ $0.5 R_{g}$. Each succeeding subgrid has its cell size increased by a factor of 2 , and so the outermost subgrid has $\Delta_{5}=8 R_{g}$ and covers a cube of size $256 R_{g} \times 256 R_{g} \times 256 R_{g}$. In practice, we used only a quarter of the full cubic domain, by focusing on a $90^{\circ}$ wedge around the $z$-axis. Thus, we employed $32 \times 32 \times 64$ cells along $x y z$ and used periodic boundary conditions in the azimuthal direction.

We define the quantities $\rho, \rho v$ and $\rho \epsilon$ at the centers of cubic cells and the components of $\boldsymbol{B}$ at the corresponding cell edges. By using continuous piecewise parabolic approximations of $\boldsymbol{v}$ and $\boldsymbol{B}$ when solving equation (7), the code

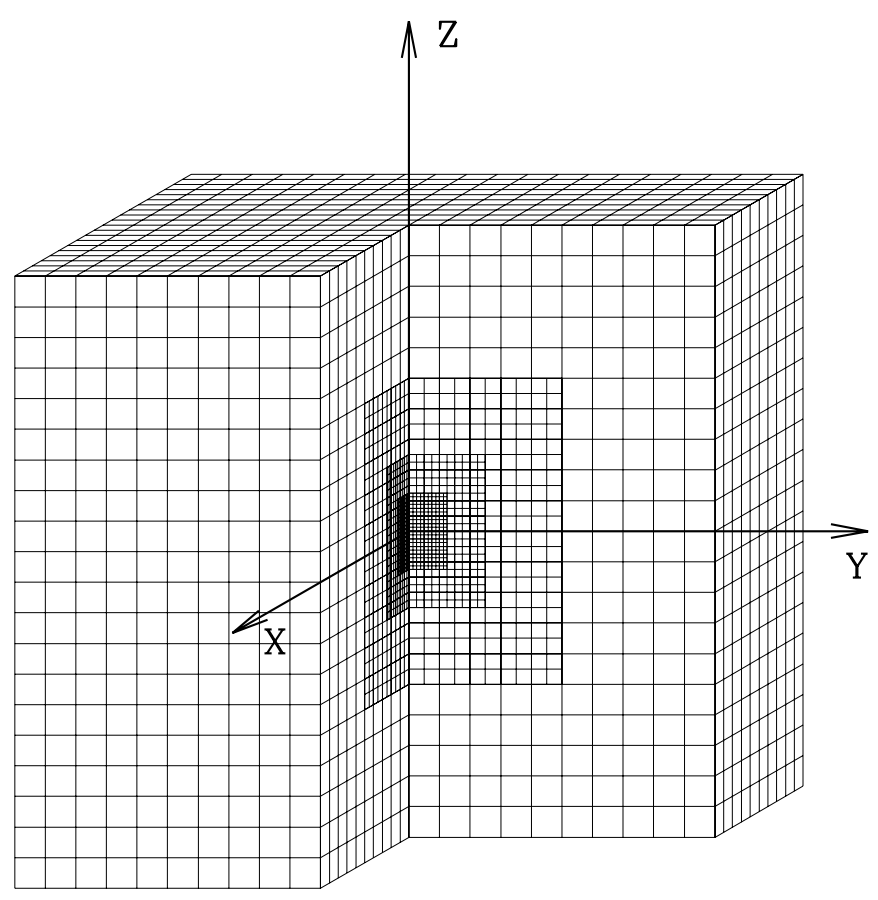

Fig. 1.- Schematic view of the nested Cartesian grid used in the simulations. The example shown has four subgrids, each of size $20 \times 20 \times 20$. In the actual calculations, five subgrids were used with dimensions $64 \times 64 \times 64$. The black hole is located at the origin. 
avoids the "explosive" instability that is found in MHD codes based on the original MOC algorithm (Clarke 1996).

The energy equation in numerical MHD needs to be handled with care. Because of the finite spatial resolution, field lines can reconnect, leading to a loss of magnetic energy without a compensating increase in the internal energy of the gas. This leads to uncontrolled energy loss in the simulation (e.g., Stone \& Pringle 2001; Igumenshchev \& Abramowicz 2001).

To fix the problem, we introduce an explicit artificial resistivity $\eta$ and set its magnitude to be larger than the effective numerical resistivity associated with numerical reconnection. Following Stone \& Pringle (2001), we choose

$$
\eta=\eta_{0} \frac{|\boldsymbol{\nabla} \times \boldsymbol{B}|}{\sqrt{4 \pi \rho}} \Delta^{2},
$$

where $\eta_{0}$ is a dimensionless parameter and $\Delta$ is the grid spacing. The magnetic Reynolds number corresponding to this resistivity is

$$
\operatorname{Re}_{m} \simeq \frac{1}{\eta_{0}}\left(\frac{L}{\Delta}\right)^{2},
$$

where $L$ is the characteristic spatial scale of the problem.

Ryu, Jones, \& Frank (1995) have shown that the effective Reynolds number due to numerical resistivity has the same functional dependence on $L$ and $\Delta$ as in equation (9), and they estimate the corresponding coefficient $\left(\eta_{0}\right)_{n}$ to be in the range $0.2-0.5$ for their second-order MHD code based on the total variation-diminishing scheme. We expect that our code has the same or somewhat lower numerical resistivity. For this reason, we have used $\eta_{0}$ in the range of 0.3-0.5. Test runs show a significant improvement in energy conservation when we choose $\eta_{0}$ in this range.

In principle, the nonuniform nested numerical grid (Fig. 1) could introduce perturbations into the flow at the interfaces between subgrids because of differences in the numerical viscosity and resistivity on the two sides of the boundary. We checked for this effect in rotating hydrodynamic accretion flows (Igumenshchev et al. 2000) and found that the perturbations were small and had no significant effect on the global flow. The present simulations of MHD accretion flows again show only minor effects at subgrid interfaces.

\subsection{Initial and Boundary Conditions}

We initialize our simulations with a spherically symmetric flow, described by the following self-similar Bondi solution for a $\gamma=5 / 3$ gas in the Newtonian gravitational potential of a point mass $M$,

$$
\begin{gathered}
\rho(R)=\frac{\dot{M}}{4 \pi} \frac{R^{-3 / 2}}{v_{0} \sqrt{G M}}, \quad v_{R}(R)=-v_{0} \sqrt{\frac{G M}{R}}, \\
\epsilon(R)=\frac{3}{5}\left(1-\frac{v_{0}^{2}}{2}\right) \frac{G M}{R},
\end{gathered}
$$

where $\dot{M}$ is the mass accretion rate and $v_{0}$ is a dimensionless parameter that can take any value in the range $0 \leq v_{0} \leq$ $\sqrt{2}$. In all our simulations we have taken $v_{0}=1$, which corresponds to supersonic Bondi accretion with Mach number $\mathscr{M}=1.7$. We take the initial magnetic field to be uniform with only one nonzero component: $B_{z}$. We specify the initial strength of the magnetic field $B_{z}$ by a parameter $b_{0}$ defined as follows:

$$
\frac{B_{z}^{2}}{8 \pi}=b_{0}^{2} \frac{G M \rho_{\text {out }}}{R_{\text {out }}},
$$

where $\rho_{\text {out }}$ is the density of the gas at the outer radius of the grid, $R_{\text {out }}=256 R_{g}$. We choose $b_{0}^{2} \ll 1$, which ensures that the magnetic field has negligible influence on the flow dynamics early in the simulation.

At the outer boundary we assume that, at all times, the density and the velocity are given by the Bondi solution (10) and the magnetic field is equal to its initial value. At the inner boundary, close to the black hole horizon, we assume absorbing conditions. Specifically, any matter that crosses the inner radius $R_{\mathrm{in}}=2 R_{g}$ is extracted from the computational domain, and the magnetic terms in the equation of motion (2) are switched off.

\section{NUMERICAL RESULTS}

We have calculated four models, A, B, C, and D, with the parameters listed in Table 1 . All the models begin with a weak magnetic field $\left(b_{0}^{2} \ll 1\right)$, which has a negligible dynamical effect on the flow. Therefore, the spherical Bondi solution with which the flow is initialized is stable and persists for some time. As the gas flows in, the strength of the magnetic field increases, with the most rapid increase occurring in the innermost region. At a certain critical time the field becomes strong enough to modify the flow dynamics.

Figure 2 shows the evolution of the mass accretion rate onto the black hole for models A (thin line), B (dashed line), and $\mathrm{D}$ (thick line). At the beginning of the simulations, the accretion rates in all three models experience a quick relaxation. This initial relaxation is due to the fact that there is a minor inconsistency between the self-similar solution (10) with which the flow is initialized and the actual problem, which has absorbing inner boundary conditions as well as a pseudo-Newtonian potential (eq. [6]). At the end of the initial relaxation, the flow takes up a slightly modified steady-state configuration, and the mass accretion rate onto the black hole becomes equal to the rate of mass input at the outer boundary. At this point, the flow is essentially the standard Bondi flow. Model D, which is a pure hydrodynamic simulation, does not change any further after this initial relaxation. Models A, B, and C, however, which have magnetic fields, undergo significant evolution.

TABLE 1

\begin{tabular}{ccc}
\multicolumn{3}{c}{ PARAMETERS OF THE MODELS } \\
\hline \hline Model & $\eta_{0}{ }^{\mathrm{a}}$ & $b_{0}{ }^{\mathrm{b}}$ \\
\hline A $\ldots \ldots \ldots \ldots$ & 0.5 & 0.3 \\
$\mathrm{~B} \ldots \ldots \ldots \ldots$ & 0.5 & 0.1 \\
$\mathrm{C} \ldots \ldots \ldots \ldots$ & 0.3 & 0.3 \\
$\mathrm{D} \ldots \ldots \ldots \ldots$ & 0 & 0 \\
\hline
\end{tabular}

a The quantity $\eta_{0}$ is a dimensionless artificial resistivity parameter, defined in eq. (8).

b The quantity $b_{0}$ characterizes the initial magnetic field and is defined in eq. (11). 


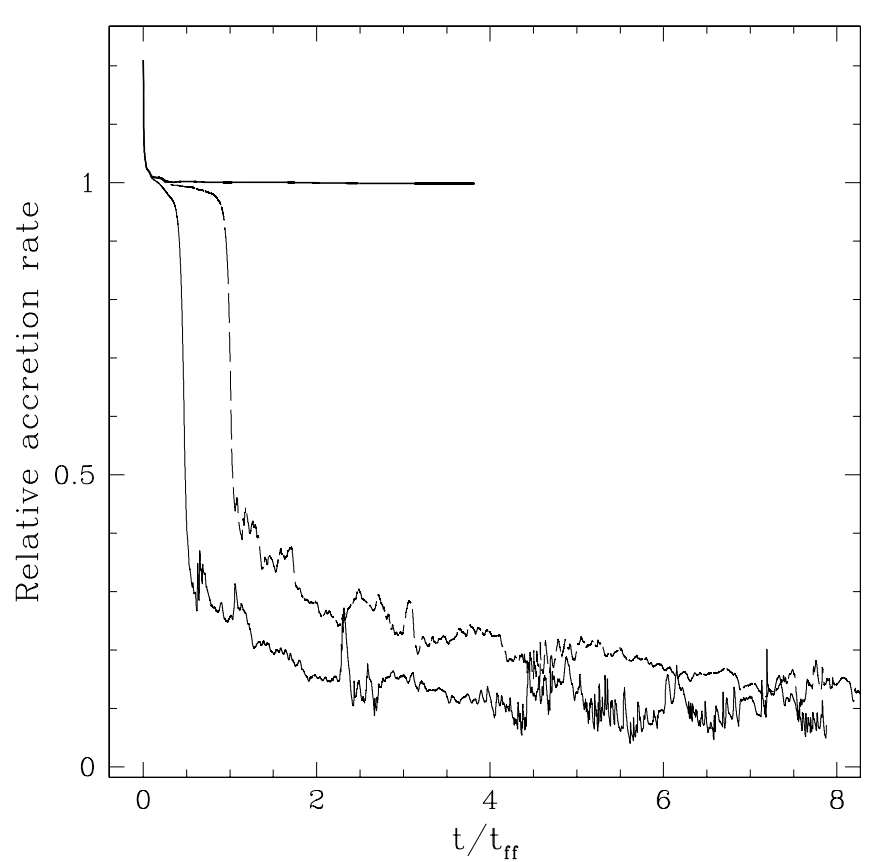

FIG. 2.-Time evolution of the mass accretion rate, normalized to the Bondi rate, in model A (thin line), model B (dashed line) and model D (thick line). Note the suppression of the accretion rate in models A and B, both of which have magnetic fields. Model D corresponds to a pure hydrodynamic flow.

Figure 3 shows the configuration of magnetic field lines in model $\mathrm{A}$ at time $t=0.5$; we measure time in units of the free-fall time from the outer radius $R_{\text {out }}$ of the computational domain. We see that the initially parallel field lines

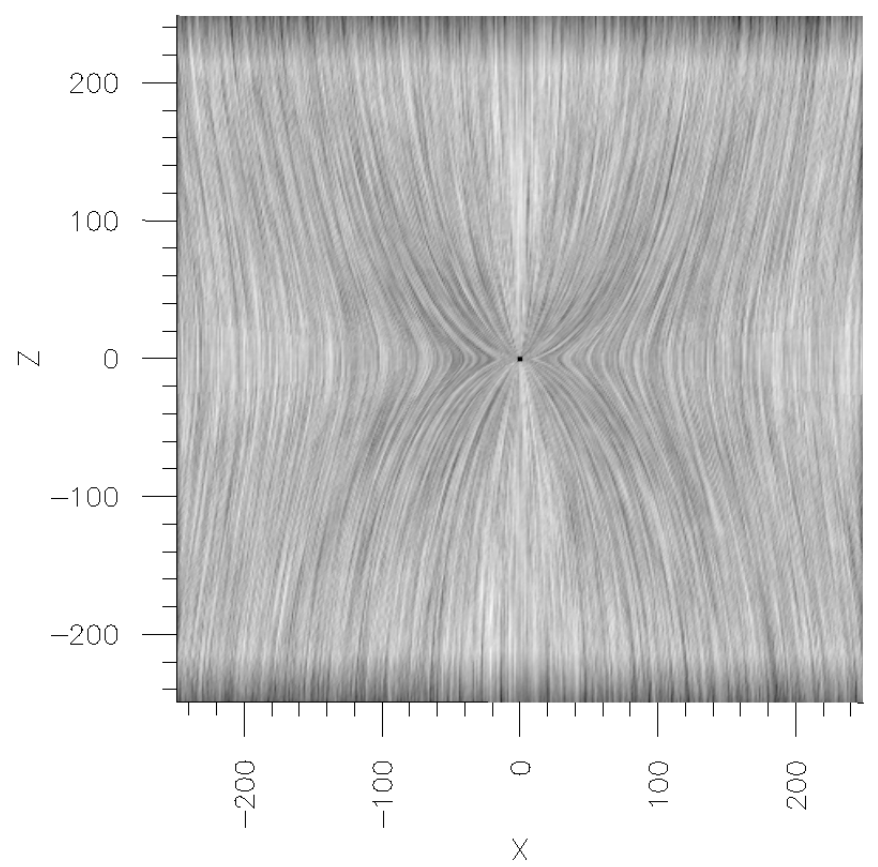

FIG. 3.-Magnetic lines in the $x-z$ plane in model A at time $t=0.5$ (measured in units of the free-fall time from the edge of the grid). The axes are labeled in units of the gravitational radius. Initially, at $t=0$, the magnetic field is uniform and in the $z$-direction. With time, the frozen-in field lines are deformed under the action of the spherically converging accretion flow and form an axisymmetric structure around the $z$-axis. The black hole is at the origin. The lines have been plotted using the line integral convolution method of Cabral \& Leedom (1993). are pulled in toward the black hole as they are swept in by the converging flow streamlines. Note that, near the "equatorial" plane, oppositely directed magnetic lines closely approach each other ("equator" and "pole" are defined with respect to the large-scale magnetic field). This leads to efficient reconnection soon after this time.

At $t \simeq 0.5$ in model $\mathrm{A}$, the strength of the magnetic field in the vicinity of the black hole reaches equipartition with the gas pressure, and the accretion rate experiences a sudden large drop. This drop represents the effect of the amplified magnetic field, which suppresses accretion in the equatorial zone and forces matter to accumulate within a "core" region in the vicinity of the black hole. The shape of the dense core is roughly toroidal in this simulation, with low-density regions along the two poles; this particular geometry is the result of the bipolar structure of the assumed large-scale field. Because the initial Bondi flow is supersonic, an MHD shock forms where the inflowing matter meets the core. The postshock gas is subsonic and sub-Alfvénic. In model B the same drop in accretion rate is again seen, except that it happens at a later time $(t \approx 1)$. This model starts with a weaker field; therefore, it takes longer for the magnetic pressure to build up to equipartition strength. Model C, with a smaller value of the resistivity parameter $\eta_{0}$, has an evolution almost identical to that of model A. All three models relax to a new state in which the mass accretion rate is several times less than the mass supply rate. The accretion rates in models A, B, and C continue to evolve slowly with time, and by the end of the simulations $(t \sim 8)$, the rates are roughly an order of magnitude less than the mass supply rate. The accretion rates are highly variable, however, reflecting the unstable nature of the flows. All three models have very similar final states.

Here we discuss model $\mathrm{A}$ in detail as a representative example. As already mentioned, a dense core is formed in the innermost region of the accretion flow once the magnetic field reaches equipartition with the gas pressure. The core is bounded by a quasi-spherical shock. The size of the core and the mass contained in it increase with time because of the accumulation of matter; matter flows onto the core from the outside at a rate determined by the rate of mass input at the outer boundary, while mass flows out of the core into the black hole at a much lower rate (roughly a factor of 10 lower).

Figures 4, 5, and 6 show the distribution of density, magnetic field lines, and velocity streamlines, respectively, in the core region at a relatively late time, $t=7.88$. The influence of the large-scale magnetic field introduced through the outer boundary condition is still visible in the polar direction. The magnetic field here is almost radial and has its maximum strength. Correspondingly, the density takes its minimum value, so that the plasma $\beta \equiv P_{g} / P_{m}$ is quite small, $\beta \sim 0.1$. The accreting matter moves along the magnetic lines with supersonic velocity; the velocity is roughly of order the Alfvén speed. Because of the high velocity there is no accumulation of matter.

The picture is very different in the equatorial region-see Figures 4-6. The topology of the magnetic field is much more tangled, and the gas is denser. The accumulated matter is highly inhomogeneous, with filaments of higher and lower density being sandwiched between each other (Fig. 4). The inhomogeneous structure is the result of convection in the core driven by the heat released during episodes of magnetic reconnection. We find that dissipation 


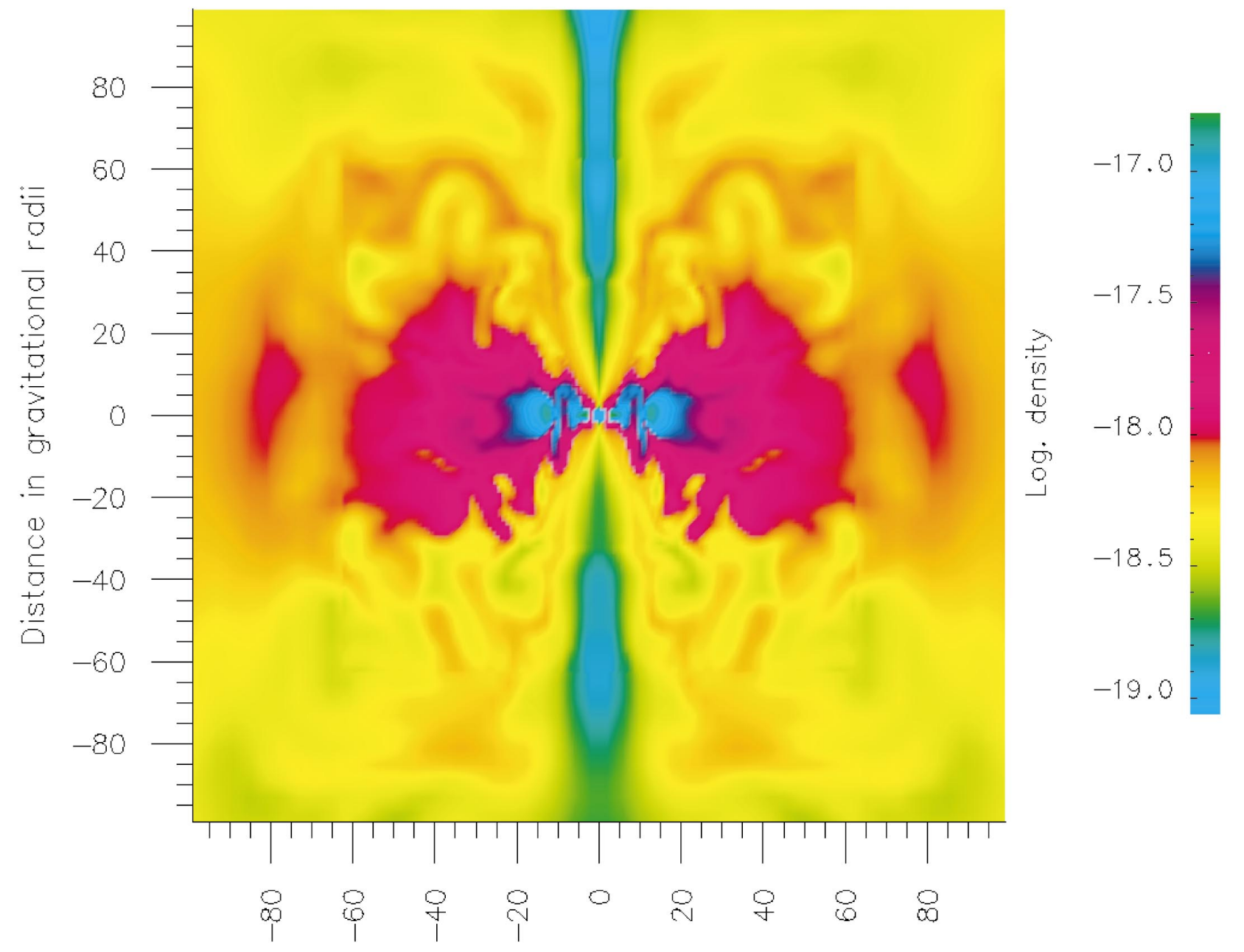

FIG. 4.-Density distribution in the $x-z$ plane in model A at time $t=7.88$. The black hole is located at the origin. Matter is concentrated toward the equatorial plane (the horizontal axis) and toward the black hole. Note the density inhomogeneities that have been produced by the motion of convective blobs. There is a shock at around $80 R_{g}$, where the supersonically infalling gas from the outer boundary meets the convective core. The two polar funnels are filled with low-density matter.

due to reconnection is a much more important heating mechanism than dissipation due to shocks and is the primary agent responsible for the development of convection in the models.

The first reconnection event occurs at a time slightly later than that shown in Figure 3. The reconnection and corresponding energy release happens exactly at the equatorial plane, in the vicinity of the inner boundary, where the magnetic field is strongest and where oppositely directed field lines approach each other most closely. After the reconnection event, the local strength of the magnetic field is reduced to a subequipartition level (as visualized by Shvartsman 1971), and the gas is heated by the energy released in the reconnection. The heated gas expands and forms a convective blob that moves outward as a result of buoyancy force. During the motion of the hot blob through the ambient medium, it deforms magnetic lines and causes new reconnection events. This leads to the formation of other hot blobs, which again become convective. As a result, the convective motions are self-sustained and lead to turbulence in the core. The magnetic field in the convection zone is on average close to equipartition strength, with $\beta \simeq 1-10$.

Figure 5 shows the tangled magnetic field configuration after the convection has become fully developed, and Figure 6 shows the chaotic velocity streamlines. We see numerous vortices and circulation patterns. The most efficient convection occurs at intermediate angles between the poles and the equator. Somewhat less efficient convective motions are present in the equatorial plane, and there is no convection in the polar regions.

Note that the convective motions are not accompanied by a significant mass outflow or wind. Thus the flow does not resemble the models of Blandford \& Begelman (1999) and Das (2000), who consider powerful outflows. The discrepancy is not surprising since those authors include additional ingredients such as angular momentum and viscosity (Blandford \& Begelman; see also Narayan \& Yi 1994) and pair physics (Das).

\section{SELF-SIMILAR SOLUTIONS}

To better understand the physics of radiatively inefficient magnetized spherical accretion, we consider here a steady radial flow and seek self-similar solutions of the equations. By the assumption of self-similarity, we expect the plasma $\beta$ to be independent of radius $R$. Hence, we write the gas, 


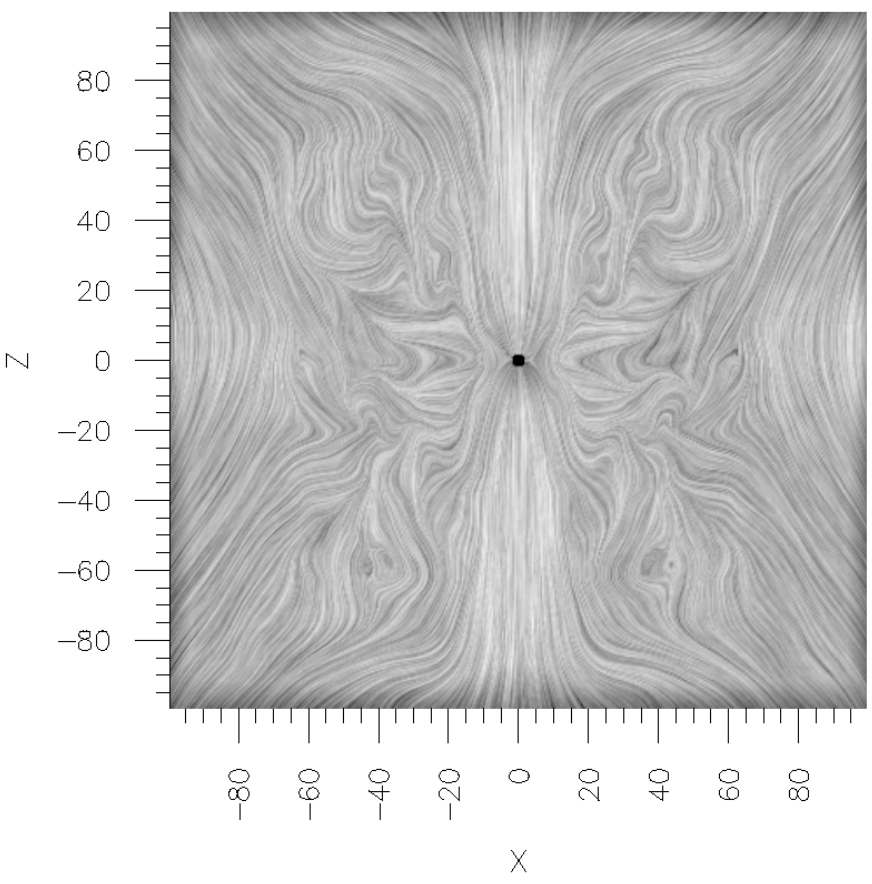

Fig. 5.-Magnetic field lines in the $x-z$ plane in model A at time $t=7.88$. The component of field lines parallel to the plane is shown. Except for the polar regions, the magnetic field elsewhere has a highly tangled morphology. This is the result of convection.

magnetic, and total pressure as

$$
P_{g} \equiv \rho c_{s}^{2}, \quad P_{m}=\frac{1}{\beta} \rho c_{s}^{2}, \quad P_{\mathrm{tot}}=P_{g}+P_{m}=\frac{\beta+1}{\beta} \rho c_{s}^{2},
$$

where $c_{s}$ is the isothermal sound speed of the gas. The radial

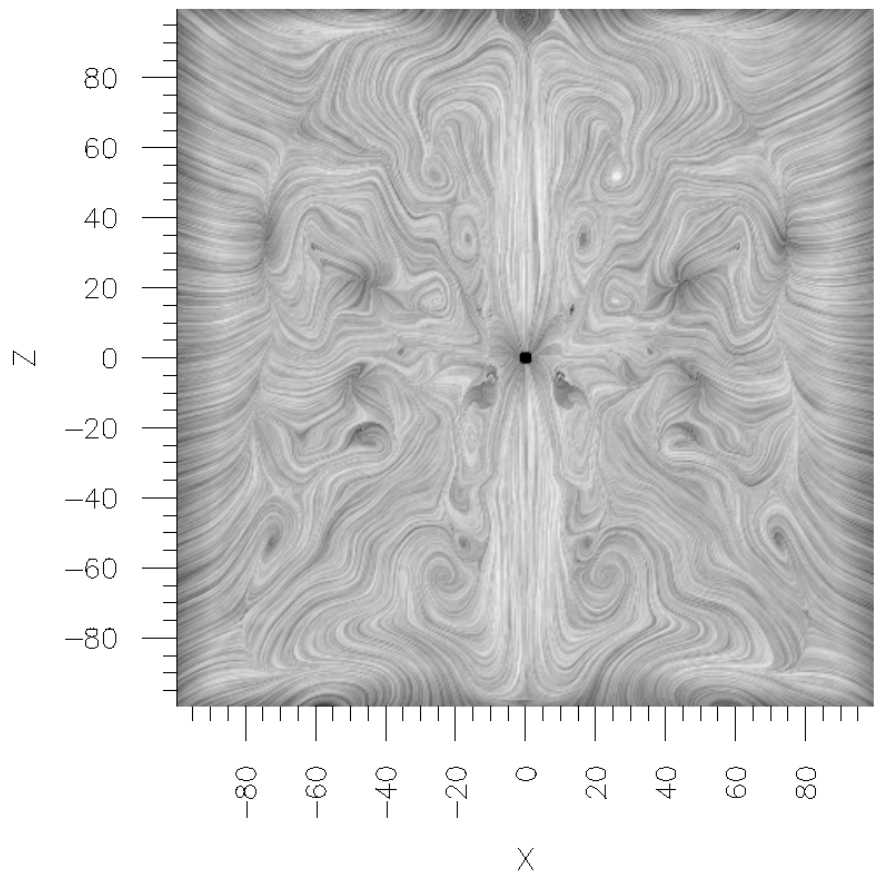

Fig. 6.-Velocity streamlines in the $x-z$ plane in model $\mathrm{A}$ at time $t=7.88$. The component of streamlines parallel to the plane is shown. A complicated pattern is seen, with numerous vortices and eddies. This is the result of convection. momentum equation and the gas energy equation take the form

$$
\begin{gathered}
\frac{d}{d R}\left(\frac{v_{R}^{2}}{2}\right)=-\frac{1}{\rho} \frac{d P_{\mathrm{tot}}}{d R}-\frac{G M}{R^{2}}, \\
\rho v_{R} T \frac{d s}{d R} \equiv \rho v_{R}\left(\frac{1}{\gamma-1} \frac{d c_{s}^{2}}{d R}-\frac{c_{s}^{2}}{\rho} \frac{d \rho}{d R}\right) \\
=-\frac{1}{R^{2}} \frac{d}{d R}\left(R^{2} F_{c}\right)+Q_{\mathrm{diss}},
\end{gathered}
$$

where $v_{R}$ is the radial velocity (assumed negative for accretion), $s$ is the specific entropy of the gas, $\gamma$ is the adiabatic index, and $F_{c}$ is the outward flux of energy due to convection. The term on the left-hand side of equation (14) describes the inward advection of energy, the first term on the right-hand side is the divergence of the convective energy flux, and $Q_{\text {diss }}$ is the rate of heating of the gas by dissipation.

Following Narayan \& Yi (1994; see also Kato, Fukue, \& Mineshige 1998; Narayan et al. 2000), we use a simple parametric form to represent the convective flux,

$$
F_{c}=-\alpha_{c} c_{s} \operatorname{R} \rho T \frac{d s}{d R},
$$

where $\alpha_{c}$ is a dimensionless constant. For the heating term, $Q_{\text {diss }}$, we note that there are at least two sources of dissipation: (1) energy release through magnetic reconnection and (2) viscous and resistive dissipation at small scales as a result of a turbulent cascade. It is not possible to model these processes in detail. Instead, we note that the ultimate source of energy is the gravitational potential energy of the accreting gas, and so we write

$$
Q_{\mathrm{diss}}=-\alpha_{d} \frac{v_{R}}{R} \rho \frac{G M}{R},
$$

where $\alpha_{d}$ is another dimensionless constant (the negative sign is because $v_{R}<0$ ).

We consider self-similar flows in which the various variables behave as power laws in radius (see the analogous discussion of CDAFs in Narayan et al. 2000),

$$
\begin{gathered}
c_{s}(R)=c_{0} v_{\mathrm{K}} \propto R^{-1 / 2}, \\
\rho(R)=\rho_{0} R^{-a}, \\
v_{R}(R)=\frac{\dot{M}}{4 \pi R^{2} \rho}=-v_{0} v_{\mathrm{K}}\left(\frac{R_{g}}{R}\right)^{3 / 2-a} \propto R^{-2+a},
\end{gathered}
$$

where $v_{\mathrm{K}}=(G M / R)^{1 / 2}$ is the Keplerian velocity and $a$ is a power-law index that takes one of two values, $\frac{3}{2}$ or $\frac{1}{2}$ (see Narayan et al. 2000; Quataert \& Gruzinov 2000). The coefficients $c_{0}$ and $v_{0}$ are dimensionless coefficients whose values are determined by substituting the solution into the two conservation equations (13) and (14). The coefficient $\rho_{0}$ is proportional to the accretion rate $\dot{M}$ and scales out of the problem.

The simplest case to consider is the pure hydrodynamic problem, in which gas with $\gamma=5 / 3$ accretes onto a black hole, with neither convection nor dissipative heating. The self-similar solution with $a=3 / 2$ automatically satisfies the energy equation. Substituting the solution in the momentum equation gives

$$
v_{0}^{2}=2-\frac{5(\beta+1)}{\beta} c_{0}^{2} .
$$


We thus have a family of solutions in which $v_{0}$ is a free parameter and $c_{0}$ is determined by equation (18) (with $\beta \rightarrow \infty$ since there is no magnetic field),

$$
c_{0}^{2}=\frac{1}{5}\left(2-v_{0}^{2}\right) .
$$

This is the self-similar solution given in equation (10), which was used to initialize the numerical simulations. The same self-similar solution is often used even for magnetized spherical accretion, under the assumption that the field will achieve equipartition and thereby have a self-similar scaling. But this is not correct. When there is a magnetic field, there is bound to be reconnection (see the discussion in $\S 1$ ), and this means that the entropy of the gas will increase inward. This is not consistent with the assumed scaling, $a=3 / 2, \gamma=5 / 3$, which corresponds to a constant entropy (Quataert \& Narayan 1999).

In the presence of magnetic fields, we need to consider the more general problem with finite values of $\beta, \alpha_{c}$, and $\alpha_{d}$ and a general value of $\gamma$. Let us first assume that $a$ takes the standard Bondi value of $\frac{3}{2}$. In this case, all the terms in the energy equation (14) are of the same order (all scale as $R^{-4}$ ), and the energy equation gives the following relation between $v_{0}$ and $c_{0}$,

$$
\left(\frac{1}{\gamma-1}-\frac{3}{2}\right)\left(v_{0}-\alpha_{c} c_{0}\right) c_{0}^{2}=\alpha_{d} v_{0}
$$

This relation, combined with equation (18) from the momentum equation, allows us to solve uniquely for $v_{0}$ and $c_{0}$ for given values of $\beta, \alpha_{c}, \alpha_{d}$, and $\gamma$. However, not all combinations of these parameters lead to a physical solution. For instance, consider the case when $\alpha_{c} c_{0} \ll v_{0}$. Equation (20) then gives

$$
c_{0}^{2}=\alpha_{d} /\left(\frac{1}{\gamma-1}-\frac{3}{2}\right),
$$

which shows that $\gamma$ has to be less than $\frac{5}{3}$ if $c_{0}^{2}$ is to be positive and finite. In fact, the constraint on $\gamma$ is even more severe. This can be seen by substituting equation (21) in equation (18) and solving for $v_{0}^{2}$. The requirement that $v_{0}^{2}>0$ leads to

$$
\frac{1}{\gamma-1}>\frac{3}{2}+\frac{5(\beta+1) \alpha_{d}}{2 \beta},
$$

which gives an upper limit on $\gamma$ that is smaller than $\frac{5}{3}$.

The reason for these constraints is that the value $\gamma=5 / 3$ is a singular case for a self-similar solution with $a=3 / 2$. This fact was noted by Quataert \& Narayan (1999) for the case of a rotating viscous flow, but the same argument applies here as well. When the condition $\alpha_{c} c_{0} \ll v_{0}$ that was used to derive equation (21) is not satisfied, the inequality (22) becomes modified to a more complicated relation. However, the qualitative features remain the same; namely, $\gamma$ has to be smaller than a certain value (which is less than $\frac{5}{3}$ ) in order to have a physical solution.

Let us next consider a self-similar solution with $a<3 / 2$. Now, the various terms in the energy equation are no longer of the same order. The entropy advection term and the heating term still vary as $R^{-4}$, but the term describing the divergence of the convective flux varies as $R^{-5 / 2-a}$. The latter term dominates at large $R$. Since there is no other term to balance this term, the only way to satisfy the energy equation is to ensure that its coefficient is equal to zero. This requires $a=1 / 2$.
Let us therefore set $a=1 / 2$. As we have just argued, the energy equation is automatically satisfied (to leading order) at large $R$. The momentum equation also becomes simpler since the term involving $v_{R}$ is smaller than the other two terms and may be neglected. Thus we find

$$
c_{0}^{2}=\frac{2 \beta}{3(\beta+1)} .
$$

The value of the parameter $v_{0}$ is not uniquely determined by this analysis but is fixed by boundary conditions. In particular, close to the black hole, where the various terms in the energy equation become of comparable order (in fact, the convective term probably becomes less important than the other two terms), the flow will make a transition to a different regime; this is also the region where the flow matches onto the absorbing boundary condition at the black hole. We do not discuss the physics of this region since it is beyond the scope of the present paper.

A feature of the $a=1 / 2$ self-similar solution is that $\gamma=5 / 3$ is not a singular case (the solution is singular when $\gamma=3$, but this has no practical consequences). Thus, for an accretion flow with $\gamma=5 / 3$, the $a=1 / 2$ solution appears to be more robust than the $a=3 / 2$ solution. For lower values of $\gamma$, both the $a=3 / 2$ solution and the $a=1 / 2$ flow may be allowed, and it is not a priori obvious which solution would be picked by nature. We suspect that the $a=1 / 2$ solution is the flow of choice under most conditions (see also the discussion of the $a=1 / 2$ law by Gruzinov 2001).

The two solutions discussed above are very different from each other. In the $a=3 / 2$ flow, convection is a relatively minor perturbation and the energy balance is primarily between energy advection and dissipative heating. This flow is very similar to the standard Bondi accretion flow, with only minor changes in the values of some coefficients. In contrast, in the new $a=1 / 2$ solution, the convective flux dominates the energy equation. We therefore refer to it as a convection-dominated Bondi flow (CDBF). This flow deviates remarkably from the standard Bondi solution; in fact, it resembles the CDAF solution.

The CDBF has a steady outward flux of energy due to convection. The convective luminosity is

$$
L_{c}=4 \pi R^{2} F_{c}=\frac{\alpha_{c} c_{0}^{3}}{2 v_{0}}\left(\frac{1}{\gamma-1}-\frac{1}{2}\right) \dot{M} c^{2} \equiv \varepsilon_{c} \dot{M} c^{2} .
$$

Thus, convection transports a fraction of the binding energy of the accreting gas outward. The efficiency of this process, described by the coefficient $\varepsilon_{c}$, depends on details of the flow that are not easy to determine from first principles. In the case of a CDAF, where again there is an analogous relation, numerical simulations by Igumenshchev \& Abramowicz (2000; see also Narayan et al. 2000; Igumenshchev et al. 2000) give $\varepsilon_{c} \sim 0.001-0.01$. A CDBF probably has a similar efficiency, but this needs to be checked with more detailed simulations.

\section{DISCUSSION}

\subsection{Underlying Physics and Caveats}

The principal physical effects operating in our numerical simulations may be understood by considering the energetics of the accreting magnetized plasma. We can identify three major stages in which the gravitational binding energy of the accreting gas is transformed into other forms 
of energy:

(gravitational energy)

$\downarrow$
(magnetic energy)
$\downarrow$
(thermal energy)
$\downarrow$

(convective turbulent energy, leading to convective energy transport) .

The first and second transformations, namely (gravitational energy) $\rightarrow$ (magnetic energy) and (magnetic energy) $\rightarrow$ (thermal energy) are well known and have been widely discussed (Shvartsman 1971; Bisnovatyi-Kogan \& Ruzmaikin 1974; Mészáros 1975; and numerous later papers [see $\S 1]$ ). These two processes build up the magnetic field to nearequipartition strength and modify the thermal state of the gas (and its radiative properties) relative to the standard Bondi flow. However, they have little effect on the overall dynamics of the flow.

The third transformation, (thermal energy) $\rightarrow$ (convective turbulence), does have a very important effect on the dynamics, and this appears to have been overlooked in previous studies. The importance of convection is not because it creates turbulent kinetic energy (which is just another form of pressure, like magnetic and thermal pressure), but because it causes energy transport. Convection efficiently transports energy from the deep interior of the flow, where the bulk of the gravitational energy is released, to the outer regions of the flow. Away from the center, the convective flux dominates the physics and thus has a significant effect on the structure of the flow.

The most important consequence of the presence of convection is that the mass accretion rate onto the black hole is reduced significantly compared to a standard Bondi flow with the same outer boundary conditions (of density and sound speed). In the simulations presented here, the reduction is by a factor of about 10 . The reduction occurs because of the formation of the convective core. The mean accretion velocity in the core is highly subsonic and much smaller than the radial velocity in an equivalent Bondi flow. Only very close to the inner absorbing boundary does the accretion become supersonic, in contrast to a standard Bondi flow, which has supersonic infall over a wide range of radius. The radial density profile is also very different from the $R^{-3 / 2}$ profile found in a Bondi flow.

We should caution that, in our models, we have assumed that the external medium has a uniform magnetic field. This leads to a large departure from spherical symmetry in the accretion flow at late times. For instance, Figures 4-6 show a toroidal structure in the density and a bipolar structure in the magnetic field, with two low-density high-field "poles" (oriented parallel to the external field) along which there is preferential accretion. The situation that we have simulated would be realized if the coherence scale of the field in the external medium is larger than the accretion radius $R_{a}$ - the radius at which the gas from the external medium is captured by the gravitational pull of the accreting mass. However, one could visualize other situations in which the field is tangled on small scales in the external medium, so that the accreting gas has several distinct magnetic loops.
We have tried numerical experiments on accretion flows with small-scale magnetic field of different configurations. However, we lacked sufficient numerical resolution for these experiments; the field underwent resistive dissipation before it could reach equipartition with the gas pressure. This demonstrates the importance of having adequate numerical resolution. We estimate that to conduct any believable experiments with a nonuniform external field, we will need to increase the numerical resolution by a factor of at least 2-3.

Despite the above cautionary comment, the basic physical ideas that we have presented should be valid whatever be the topology of the field; namely, line stretching and field amplification lead to reconnection, which leads to gas heating, which leads to convection. This chain of argument requires merely that the magnetic field should be frozen into the gas and that the magnetic field should build up to equipartition strength before the gas falls into the black hole. Since the magnetic pressure grows as $R^{-4}$ whereas the gas pressure varies only as $R^{-5 / 2}(\S 1)$, the latter condition should be easily satisfied in most cases of interest.

Another cautionary comment is related to the fact that our simulations have not reached steady state. The convective core grows slowly and has reached a size of only about $80 R_{g}$ by the end of our simulations. This is still a factor of 3 smaller than the size of the grid. In a real accretion flow we imagine that the convective core would grow until it extends beyond $R_{a}$. The convective flux would then flow out into the external medium and perhaps modify the medium in the vicinity of $R_{a}$. Ultimately, a steady state should result, but the present simulations have not been run long enough to determine the nature of the steady state.

\subsection{Self-similar Convection-dominated Bondi Flow}

In $\S 4$ we have presented a simplified set of equations to describe spherical accretion of a magnetized plasma. The equations include two critical pieces of physics: (1) heating of the gas by reconnection (and other dissipative processes) and (2) convective energy transport. Depending on parameters, we find that there are two distinct self-similar solutions of the equations.

One solution is similar to the standard Bondi solution; the density varies as $\rho \propto R^{-3 / 2}$ and the velocity varies as $v_{R} \propto R^{-1 / 2}$. This solution is possible whenever convection is not very strong and when the adiabatic index $\gamma$ of the gas is smaller than a limit which is less than the standard value of $\frac{5}{3}$ (see discussion below eqs. [21] and [22]). For given boundary conditions at the accretion radius $R_{a}$, the mass accretion rate in this solution is similar to the Bondi accretion rate $\dot{M}_{\text {Bondi }}$. Our numerical simulations, however, are not consistent with this solution. At this time we are not sure if the solution is relevant for any radiatively inefficient spherical accretion flow with strong fields.

The second solution is completely different from the Bondi solution. It has density varying as $\rho \propto R^{-1 / 2}$ and velocity varying as $v_{R} \propto R^{-3 / 2}$. The scalings may be understood as follows.

The bulk of the energy generation in the accretion flow happens close to the black hole. Some fraction of this energy is transported outward by convection. At radii greater than a certain transition radius $R_{\mathrm{in}}$, whose value is uncertain but is probably no more than a few $R_{g}$ or tens of $R_{g}$, the convective luminosity $L_{c}$ completely dominates over any local energy generation. Thus, for $R>R_{\mathrm{in}}$, we expect 
$L_{c}$ to be practically independent of $R$; equivalently, the convective flux $F_{c}$ must vary as $R^{-2}$. Because the accretion flow is assumed to be radiatively inefficient, the gas is virial and has a sound speed $c_{s} \sim v_{\mathrm{K}}$ (the Kepler or free-fall velocity). Thus, there is only one velocity in the problem, namely, $v_{\mathrm{K}} \propto R^{-1 / 2}$; therefore, the convective flux has to take the form $F_{c} \sim \rho c_{s}^{3} \sim \rho v_{\mathrm{K}}^{3} \sim \rho R^{-3 / 2}$. Requiring the convective flux to vary as $R^{-2}$ means that $\rho$ must scale as $R^{-1 / 2}$. Mass conservation, $\dot{M}=-4 \pi R^{2} v_{R} \rho=$ constant, then immediately gives $v_{R} \propto R^{-3 / 2}$. Thus, the structure of the flow is determined uniquely once we assume (1) that there is a significant flux of energy outward due to convection and (2) that there is no significant radiative cooling.

Because of the important role played by convection, we refer to this kind of flow as a convection-dominated Bondi flow. The scalings sketched out above allow us to estimate the mass accretion rate in this flow. In the standard Bondi problem, where a mass $M$ is embedded in a homogeneous medium of density $\rho_{\infty}$ and sound speed $c_{\infty}$, the accretion radius is given by $R_{a} \sim G M / c_{\infty}^{2}$; this is the radius at which the gravitational free-fall velocity is equal to $c_{\infty}$. The gas in the Bondi solution flows in at essentially the free-fall velocity for $R \lesssim R_{a}$; therefore, the mass accretion rate is given by $\dot{M}_{\text {Bondi }} \sim 4 \pi R_{a}^{2} \rho_{\infty} c_{\infty}$. In a CDBF, we expect $v_{R}$ to be roughly equal to the free-fall velocity at $R \sim R_{\text {in }}$. Since $v_{R}$ falls off as $R^{-3 / 2}$, this means that at $R \sim R_{a}, v_{R}$ is smaller than the local free-fall velocity by a factor $\sim R_{\text {in }} / R_{a}$. We then estimate that

$$
\dot{M}_{\mathrm{CDBF}} \approx \frac{R_{\mathrm{in}}}{R_{a}} \dot{M}_{\mathrm{Bondi}} .
$$

This shows that convection can have a profound effect on the mass accretion rate in a magnetized spherical accretion flow.

What happens to the convective luminosity when it reaches the radius $R_{a}$ ? From equations (24) and (25) we see that the convective luminosity is

$$
L_{c}=\epsilon_{c} \dot{M}_{\mathrm{CDBF}} c^{2} \sim\left(\epsilon_{c} \frac{R_{\mathrm{in}}}{R_{g}}\right) 4 \pi R_{a}^{2} \rho_{\infty} c_{\infty}^{3} .
$$

The factor within parentheses is likely to be less than unity. Therefore, the external gas should be able to transport the energy outward beyond $R_{a}$. The transport may be by convection or some related form of turbulence or by thermal conduction. It would be of interest to compute selfconsistent idealized global models including the external medium to see how much the medium is modified in the vicinity of $R_{a}$ by the outward energy flux.

The convective core region in the simulations presented in this paper (Figs. 4, 5, and 6) has features that are qualitatively similar to the predictions of the self-similar CDBF solution. Unfortunately, because of limited spatial resolution, we have not been able to make quantitative comparisons between the numerical results and the predictions of the analytical model. The averaged radial profiles of density, velocity, gas, and magnetic pressures show significant oscillations, both in space and time. They also show the influence of the inner boundary (black hole) and outer boundary (the shock where the free-falling gas meets the convective core), which cannot be separated out because of the relatively small range of radius covered by the simulations. More extensive numerical work is required to confirm the theoretical predictions in detail. Also, in a real flow, we expect the CDBF zone to extend all the way out to $R_{a}$, where it would match onto the ambient density. The simulations have not yet reached this stage.

Nevertheless, we attempt a crude comparison between the simulations and the analytical CDBF solution. At the end of the various runs, the central core region in our numerical simulations has a radius of about $R_{\text {core }} \sim 80 R_{g}$. Given that we are in a non-steady state situation, we expect that $R_{\text {core }}$ serves the same role as the accretion radius $R_{a}$ for a steady flow. Assuming that $R_{\text {in }} \sim 10 R_{g}$, we then estimate from equation (25) that the mass accretion rate onto the black hole should be $\sim R_{\text {in }} / R_{\text {core }} \sim 1 / 8$ of the (Bondi) mass supply rate at $R_{\text {core }}$. Figure 2 shows that the accretion rate is indeed of this order.

We should note the close analogy between the CDBF solution described here and the viscous rotating (nonmagnetic) CDAF solution (Narayan et al. 2000; Quataert \& Gruzinov 2000). The CDAF is more complex because, in addition to the radial momentum equation and the energy equation, it is also strongly influenced by the angular momentum equation. In particular, there is a competition between viscosity and convection in the angular momentum balance, which plays an important role in determining the structure of the solution. Nevertheless, the particular radial scalings seen in a CDAF, $\rho \propto R^{-1 / 2}, v_{R} \propto$ $R^{-3 / 2}$, are identical to those found in a CDBF, and they result from the same physics identified above, namely, the presence of an energetically dominant convective flux and the absence of radiative cooling. Furthermore, the accretion rate in a CDAF is reduced compared to that in an equivalent advection-dominated accretion flow (ADAF; see Narayan \& Yi 1994; Abramowicz et al. 1995), for the same outer boundary conditions, by a factor $\sim R_{\text {in }} / R_{\text {out }}$, which is similar to the factor given in equation (25). The exact value of $R_{\mathrm{in}}$ in the two problems depends on the nature of the flow close to the black hole. This is discussed in a separate paper (Abramowicz et al. 2002).

\subsection{The Importance of Resistive Heating}

We have carried out a few numerical experiments to clarify the role of resistive heating in a convectiondominated Bondi flow. First, as already mentioned, when we use too large an artificial resistivity in the simulations, such that the magnetic field reconnects well before it reaches equipartition strength, we find that the flow does not make a transition to a convection-dominated form. This is not surprising since in this case there is very little heating from reconnection and therefore there is not enough entropy production to drive significant convection. Although it is not fully understood how reconnection works in astrophysical plasmas (but see the recent work of Lazarian \& Vishniac 1999), it does seem reasonable to assume that significant reconnection occurs only after the field builds up at least to equipartition strength, as proposed by Shvartsman (1971).

To further investigate the importance of resistive heating, we have carried out a series of simulations in which we set the artificial resistivity $\eta$ in equations (3) and (4) to zero. In these runs, there is significant magnetic reconnection through numerical resistivity, but there is no corresponding heating of the gas. The flows do not exhibit convection. As in models $\mathrm{A}-\mathrm{C}$ described in $\S 3$, when the magnetic field reaches equipartition with the gas pressure, a central "core" region is formed, bounded by a quasi-spherical 
shock. However, the core is more compact, and the accretion rate is only slightly reduced with respect to the Bondi rate. The flow pattern in the core is perturbed with respect to the spherical inflow because of the effect of the magnetic field, but the perturbations are not as significant as in models $\mathrm{A}-\mathrm{C}$, and the velocity streamlines are not as chaotic.

To understand how important the magnetic field is for the formation of the convective core, we have carried out three-dimensional hydrodynamic simulations with finite bulk and/or shear viscosity and with two values of $\gamma: \frac{5}{3}$ and $\frac{4}{3}$. We expected that the bulk viscosity would heat the accreting gas and that this might lead to efficient convection. Instead, we find that viscous Bondi flows are stable for a wide range of values of the bulk and shear viscosity coefficients. Combined with the experiments described in the previous paragraph, the implication is that both the electromotive forces associated with the magnetic field and the heating effect due to reconnection are important for the flow to become convection dominated; any one by itself is not enough. Once the CDBF state has been achieved, it appears to be stable and self-sustaining.

We note recent simulations of three-dimensional rotating MHD accretion flows by Machida, Matsumoto, \& Mineshige (2001) and Hawley, Balbus, \& Stone (2001). These flows exhibit the magnetorotational instability, as expected. One might expect them also to be convective, at least at large radius, and to have a radial structure similar to a CDBF (or a CDAF). Indeed, the flows obtained by Machida et al. (2001) seem to be convective and have a flattened radial density profile, close to $R^{-1 / 2}$. However, Hawley et al. (2001), who used a similar numerical technique, report that they do not observe convective motions in their models. These simulations do not include a resistive heating term, and the energy release due to (numerical) reconnection is lost from the system. (Stone \& Pringle 2001 do include an artificial resistivity, but their simulations are in two dimensions.) As noted above, when we simulate spherical accretion without including an artificial resistivity, we do not see convection.

\subsection{Astrophysical Applications}

Accretion flows in many astrophysical systems involve magnetized plasma. We would like to suggest that any astrophysical system that has radiatively inefficient spherical accretion will set up a convection-dominated flow similar to the CDBF solution discussed here and will behave very differently from the standard Bondi solution. In particular, we suggest that the mass accretion rate will be given by equation (25), which is very much less than the Bondi accretion rate. This has potentially important implications.

In a classic paper, Fabian \& Canizares (1988) used the Bondi solution to estimate the mass accretion rate $\dot{M}$ onto supermassive black holes in the nuclei of nearby giant elliptical galaxies and showed that the observed nuclear luminosities are far below the luminosity expected if the radiative efficiency is the canonical $10 \%$. The problem has become more severe in recent times with improved observations of the nucleus of our own Galaxy (Baganoff et al. 2002) and nuclei of nearby galaxies (e.g., Di Matteo et al. 2001). One solution to the luminosity problem is to assume that the accretion occurs in a radiatively inefficient mode, e.g., via an ADAF (Narayan, Yi, \& Mahadevan 1995; Fabian \& Rees 1995; Reynolds et al. 1996). The present work suggests an even simpler solution; namely, the mass accretion rate onto the supermassive black hole may be much less than the Bondi rate assumed by Fabian \& Canizares (1988). For conditions typical of galactic nuclei, say, $c_{\infty} \lesssim 10^{3} \mathrm{kms}^{-1}$, we expect $R_{a} \sim 10^{5} R_{g}$. Since $R_{\mathrm{in}}$ in equation (25) is likely to be no more than a few tens of $R_{g}$, we see that $\dot{M}$ with a CDBF could be smaller than $\dot{M}_{\text {Bondi }}$ by a large factor $\sim 10^{3}-10^{4}$.

Another application is to isolated neutron stars and black holes accreting from the interstellar medium in the Galaxy. Treves \& Colpi (1991) and Blaes \& Madau (1993) used the Bondi accretion rate to estimate the likely luminosities of accreting neutron stars and discussed the possibility of detecting them by their EUV and X-ray emission. A number of later papers have discussed theoretical predictions for the emitted spectrum (e.g., Turolla et al. 1994; Zane, Turolla, \& Treves 1996). Despite careful searches in the ROSAT all-sky survey, the predicted large number of sources has not been found (e.g., Belloni, Zampieri, \& Campana 1997). As in the case of dim galactic nuclei, we suggest that the discrepancy is because the accretion onto the neutron stars occurs via a convection-dominated flow, so that the mass accretion rate is far below the Bondi rate. There are similar implications also for accreting stellarmass black holes (e.g., Fujita et al. 1998).

Yet another possible application is to supernova explosions. In addition to the prompt collapse of a homologous core, current models of supernovae predict fallback of material over an extended period of time after the explosion (Chevalier 1989). This material, which is ejected with less than the escape speed, flows out radially, turns around at some (large) radius, and collapses back on the compact core. Some of this material may experience significant magnetic field amplification and may undergo reconnection and heating. It is then likely to develop convective motions, resulting in a reduced rate of mass fallback. This deserves further study.

\section{SUMMARY}

The main result of this paper is that radiatively inefficient spherical (i.e., nonrotating) accretion of magnetized plasma onto a compact mass has very different properties compared to spherical accretion of unmagnetized gas. The unmagnetized problem is described by pure hydrodynamics. It was solved by Bondi (1952) and has been widely applied.

Our results on the magnetized problem are based on three-dimensional numerical MHD simulations. The simulations are initialized with an analytical self-similar Bondi flow (eq. [10]) and have initially a weak, dynamically unimportant magnetic field $\left(b_{0}^{2} \ll 1\right.$; see eq. [11]). The inner boundary conditions correspond to a black hole. We find that the Bondi solution survives for a short period of time with relatively minor changes. However, during this time the magnetic field becomes progressively stronger - because of radial stretching and transverse compression of the frozen-in field lines as the gas flows in - until the magnetic pressure builds up roughly to equipartition with the gas pressure (Fig. 3).

After this time, the structure of the flow changes dramatically. The equipartition magnetic field exerts a backreaction on the free-falling gas and slows it down in the "equatorial" region. A shock forms and gas accumulates in a "core." The magnetic field begins to reconnect to main- 
tain the magnetic pressure slightly below equipartition (plasma $\beta \sim 1-10$ ). The reconnection heats the gas locally, and the resulting high-entropy material moves outward under buoyancy. The outward motion causes further stretching and amplification of the frozen-in field lines, leading to further episodes of reconnection. Before long, the plasma in the vicinity of the black hole experiences fully developed turbulent convection. With time, the convective core grows in size, as more magnetized material from the outside is added to it, while mass flows into the black hole at a much smaller rate. The flow in the convective core bears almost no resemblance to the Bondi solution (see Figs. 4, 5, and 6). One result of all this is that the accretion rate onto the black hole is reduced significantly from the Bondi rate (Fig. 2).

We have developed a simplified analytical model of a radiatively inefficient magnetized spherical flow in which heating of the gas by reconnection and convective transport of energy to large radii play an important role. The model, which we refer to as a "convection-dominated Bondi flow," is completely different from the standard Bondi model. It has density varying as $\rho \propto R^{-1 / 2}$ and velocity varying as $v_{R} \propto R^{-3 / 2}$. The mass accretion rate onto the black hole is smaller by a factor $\sim R_{\mathrm{in}} / R_{a}$ compared to the Bondi rate. Since $R_{\mathrm{in}} / R_{a} \ll 1$ for typical astrophysical objects, the accretion rate in spherical flows could be many orders of magnitude less than that given by Bondi theory. The new solution may be relevant for understanding many astrophysical phenomena, e.g., post-supernova fallback and radiatively inefficient accretion onto supermassive black holes, stellar-mass black holes, and neutron stars.

We gratefully thank Tom Abel, Marek Abramowicz, Axel Brandenburg, Eliot Quataert, and an anonymous referee for helpful discussions and comments. This work was supported by NSF grant AST 98-20686, NASA grant NAG510780, and RFBR grant 00-02-16135.
Abramowicz, M. A., Chen, X., Kato, S., Lasota, J.-P., \& Regev, O. 1995, ApJ, 438, L37

Abramowicz, M. A., Igumenshchev, I. V., Quataert, E., \& Narayan, R. 2002, ApJ, in press

Baganoff, F. K., et al. 2002, ApJ, in press

Ball, G., Narayan, R., \& Quataert, E. 2001, ApJ, 552, 221

Belloni, T., Zampieri, L., \& Campana, S. 1997, A\&A, 319, 525

Bisnovatyi-Kogan, G. S., \& Ruzmaikin, A. A. 1974, Ap\&SS, 28, 45

Blaes, O., \& Madau, P. 1993, ApJ, 403, 690

Blandford, R. D., \& Begelman, M. C. 1999, MNRAS, 303, L1

Bondi, H. 1952, MNRAS, 112, 195

Cabral, B., \& Leedom, L. 1993, Computer Graphics: Proceedings: Annual Conference Series 1993: SIGGRAPH 93 (New York: Association for Computing Machinery), 263

Chevalier, R. A. 1989, ApJ, 346, 847

Clarke, D. A. 1996, ApJ, 457, 291

Coker, R., \& Melia, F. 2000, ApJ, 534, 723

Colella, P., \& Woodward, P. R. 1984, J. Comput. Phys., 54, 174

Das, T. 2000, MNRAS, 318, 294

Di Matteo, T., Johnstone, R. M., Allen, S. W., \& Fabian, A. C. 2001, ApJ, 550, L19

Fabian, A. C., \& Canizares, C. R. 1988, Nature, 333, 829

Fabian, A. C., \& Rees, M. J. 1995, MNRAS, 277, L55

Fujita, Y., Inoue, S., Nakamura, T., Manmoto, T., \& Nakamura, K. E. 1998, ApJ, 495, L85

Gruzinov, A. 2001, preprint (astro-ph/0104113)

Hawley, J. F., Balbus, S. A., \& Stone, J. M. 2001, ApJ, 554, L49

Houck, J. C., \& Chevalier, R. A. 1991, ApJ, 376, 234

Igumenshchev, I. V., \& Abramowicz, M. A. 1999, MNRAS, 303, 309 2000, ApJS, 130, 463

2001, in AIP Conf. Proc. 586, Relativistic Astrophysics: 20th Texas Symposium, ed. J. C. Wheeler \& H. Martel (New York: AIP)

Igumenshchev, I. V., Abramowicz, M. A., \& Narayan, R. 2000, ApJ, 537, L27

Ipser, J. R., \& Price, R. H. 1977, ApJ, 216, 578

1982, ApJ, 255, 654 1983, ApJ, 267, 371

Kato, S., Fukue, J., \& Mineshige, S. 1998, Black-Hole Accretion Disks (Kyoto: Kyoto Univ. Press)
Kowalenko, V., \& Melia, F. 1999, MNRAS, 310, 1053

Lazarian, A., \& Vishniac, E. T. 1999, ApJ, 517, 700

Machida, M., Matsumoto, R., \& Mineshige, S. 2001, PASJ, 53, L1

Maraschi, L., Perola, G. C., Reina, C., \& Treves, A. 1979, ApJ, 230, 243

Maraschi, L., Roasio, R., \& Treves, A. 1982, ApJ, 253, 312

Mason, A. 1992, MNRAS, 255, 203

Mason, A., \& Turolla, R. 1992, ApJ, 400, 170

Melia, F. 1992, ApJ, 387, L25

Mészáros, P. 1975, A\&A, 44, 59

Narayan, R., Igumenshchev, I. V., \& Abramowicz, M. A. 2000, ApJ, 539, 798

Narayan, R., \& Yi, I. 1994, ApJ, 428, L13

Narayan, R., Yi, I., \& Mahadevan, R. 1995, Nature, 374, 623

Nobili, L., Turolla, R., \& Zampieri, L. 1991, ApJ, 383, 250

Ostriker, J. P., Weaver, R., Yahil, A., \& McCray, R. 1976, ApJ, 208, L61

Paczyński, B., \& Wiita, J. 1980, A\&A, 88, 23

Park, M.-G., \& Ostriker, J. P. 1989, ApJ, 347, 679

Quataert, E., \& Gruzinov, A. 2000, ApJ, 539, 809

Quataert, E., \& Narayan, R. 1999, ApJ, 516, 399

Reynolds, C. S., Fabian, A. C., Cellotti, A., \& Rees, M. J. 1996, MNRAS, 283,873

Ryu, D., Jones, T. W., \& Frank, A. 1995, ApJ, 452, 785

Scharlemann, E. T. 1981, ApJ, 246, L15

. 1983, ApJ, 272, 279

Shapiro, S. L. 1973a, ApJ, 180, 531

. 1973b, ApJ, 185, 69

Shvartsman, V. F. 1971, Soviet Astron., 15, 377

Stone, J. M., \& Norman, M. L. 1992, ApJS, 80, 791

Stone, J. M., \& Pringle, J. E. 2001, MNRAS, 322, 461

Stone, J. M., Pringle, J. E., \& Begelman, M. C. 1999, MNRAS, 310, 1002

Treves, A., \& Colpi, M. 1991, A\&A, 241, 107

Treves, A., Maraschi, L., \& Abramowicz, M. 1988, PASP, 100, 427

Turolla, R., \& Nobili, L. 1989, ApJ, 342, 982

Turolla, R., Zampieri, L., Colpi, M., \& Treves, A. 1994, ApJ, 426, L35

Zampieri, L., Colpi, M., Shapiro, S. L., \& Wasserman, I. 1998, ApJ, 505, 876

Zane, S., Turolla, R., \& Treves, A. 1996, ApJ, 471, 248

Zeldovich, Ya. B., \& Novikov, I. D. 1971, Relativistic Astrophysics (Chicago: Univ. Chicago Press) 